\title{
ORDENAMIENTO PROPUESTO COMO ALTERNATIVA AL CRECIMIENTO DEL CASCO URBANO DE PARAMONGA
}

\author{
Alberto Huiman Cruz ${ }^{\mathrm{a}}$
}

\section{RESUMEN}

El Casco Urbano de Paramonga, se ubica en la parte oeste del distrito del mismo nombre. Tiene importancia histórica como centro agroindustrial del norte chico, que le ha valido un acelerado pero desordenado crecimiento; por ello, la presente investigación plasma el pasado, presente y la propuesta de ordenamiento urbano al 2015. Esto se logra a través del acopio de datos existentes y de la información técnicamente generada, seguido de la depuración, digitación y digitalización del nuevo grupo, con el respectivo análisis de la información de tipo teórica y gráfica, para su posterior representación en un documento.

La articulación, los Servicios Básicos (agua para consumo humano, desagüe, energía eléctrica, y la recolección, acopio y tratamiento de residuos sólidos), la dinámica poblacional, la vivienda, la salud, la educación, la gestión municipal, y las actividades económico - productivas; son los aspectos que se contemplan. De cada uno de ellos se determina la actual situación, para zonificar la ocupación del espacio físico urbano y proyectar las expectativas, según las bondades que ofrece y la urgencia de necesidades por los efectos que puede acarrear. Así, en un Plano de Propuestas de Desarrollo Urbano al 2015, se grafica la consolidación de las zonas existentes, la propuesta de nuevas áreas verdes, de servicios, y de habilitación; de acuerdo a la tendencia de demanda proyectada.

Palabras Claves: Ordenamiento Urbano, Ordenamiento, Paramonga, Casco Urbano.

\section{ABSTRACT}

The Urban Helmet of Paramonga, the west of the district of he himself name is located in the part. It has historical importance like agro-industrial center of the small north, that accelerating but disordering growth has been worth him; for that reason, the present investigation shapes the past, present and the proposal of urban ordering to the 2015 . This is obtained through storing of existing data and the technically generated information, followed of the purification, digitations and digitalization of the new group, with the respective analysis of information of the theoretical and graphical type, for its later representation in a document.

The joint, the basic services (water for human consumption, empty, electrical energy, and the harvesting, storing and treatment of solid remainders), population dynamics, the house, the health, the education, the municipal management, and the productive activities economic; they are the aspects that are contemplated. Of each one of them the present situation is determined, in order to zonificar the occupation of the urban physical space and to project the expectations, according to kindness that offers and the urgency of necessities by the effects that can carry.

Thus, in a Plane of Proposals of Urban Development to the 2015, graphical the consolidation of the existing zones, the proposal of new green areas, of services, and of rating; according to the tendency of projected demand.

Key words: Urban ordering, Ordering, Paramonga, Urban helmet.

\section{INTRODUCCION}

Paramonga es un distrito, conformado por una urbe y pequeños pueblos rurales en la parte más septentrional.

El objeto de investigación se denomina Casco Urbano de Paramonga, por ser una circunscripción del distrito con preeminencia de servicios básicos y coexistencia de actividades urbanas y rurales. No se utiliza el término ciudad, al no ajustarse a la norma de acepción técnica y por registrar tasa de crecimiento poblacional negativa en los 02 últimos periodos intercensales.

El desarrollo de la presente investigación, se divide en dos partes: El análisis de la situación actual y la propuesta, respectivamente. La primera, comienza con el diagnóstico, contiene un análisis de la situación actual del ámbito de estudio, elaborado desde diferentes enfoques, pues se conjugan los pocos documentos recopilados y recurre a diferentes fuentes de información: Revisión de bibliografía, encuesta, realización de taller participativo, entrevistas y observaciones de campo. Se realiza un acercamiento general del distrito, donde se ilustran los principales procesos y cambios sucedidos para el casco urbano; seguidamente, son analizados los aspectos sociales (dinámica poblacional, salud, educación, gestión municipal y actividades económico - productivas), y el estado de la infraestructura actual (vías de comunicación, Zviviendas y servicios básicos).

Luego, se discute y/o analiza el diagnóstico, considerando los problemas más apremiantes, por cada rubro citado, y finalmente se plasman las conclusiones y recomendaciones.

La segunda contiene la propuesta de ordenamiento urbano en sí, eliminando entelequias y recomendando soluciones y acciones a problemas básicos.

Así, partiendo del postulado 'Ordenar es poner cada cosa en su lugar y un lugar para cada cosa', se elabora una alternativa de ordenamiento urbano, acompañada de los proyectos que contribuirán a su realización y coadyuvaran con el desarrollo local; ello enmarcado en la promoción del status quo en un horizonte temporal al año 2015.

Por último, se pretende obtener un cuaderno de bitácora que proporcione los insumos necesarios para poder analizar la situación del casco urbano de Paramonga correctamente, y formular políticas duraderas con miras al desarrollo integral; de acuerdo al rol que cumple Paramonga en la provincia de Barranca.

\footnotetext{
a Ingeniero Geógrafo de la Universidad Nacional Federico Villarreal, consultor en cartografía, manejo de residuos sólidos y tratamiento de aguas residuales.
} 


\section{MÉTODO DE INVESTIGACIÓN}

La ejecución de la presente investigación se realizó a nivel detallado, desarrollando las siguientes etapas:

\section{ETAPA PRELIMINAR DE GABINETE}

En esta etapa se realizó un acopio de información de tipo temática, cartográfica, planimétrica y gráfica. Se confeccionó el itinerario de campo, que tenia como objetivo hacer un inventario urbano, a fin de microlocalizar los usos del espacio físico.

ETAPA DE CAMPO: Es esta etapa se ejecutó el itinerario de campo.

Al haberse delimitado cada sector (en plano base) y codificar cada lote, se procedió a levantar el inventario urbano.

ETAPA DE ANÁLISIS DE GABINETE: Se hizo uso de los materiales de dibujo y edición, para que la información generada en el inventario se automatice mediante la utilización de la tecnología SIG; de modo tal que, cada lote tenga características únicas de acuerdo a lo encontrado en campo. Ello permitió dar la capacidad de hacer un análisis espacial, facilitando la creación de planos temáticos (Uso Actual del Suelo Urbano y de Zonificación del Casco Urbano). Dicha información se acompaña de cuadros sobre el aspecto social y económico del casco urbano, su evolución y tendencias.

ETAPA FINAL DE GABINETE: Analizada la información, se concluyó que el casco urbano de Paramonga necesita organizarse y ejecutar mejoras sustanciales en su entorno. Por ello, se jerarquizaron las acciones que se deben adoptar en el corto, mediano y largo plazo, hasta el 2015. Esto se recopila de manera teórica y se ejemplifica, de manera gráfica en un plano.

\section{RESULTADOS}

1. VÍAS DE COMUNICACIÓN: El acceso interprovincial es posible a través de la carretera Panamericana, el diagnóstico da como resultado que sólo el $15 \%$ de la red vial vecinal se encuentra asfaltada y no existe terminal terrestre de carga ni de pasajeros. Se agudiza el problema de la carencia de vías de conexión provincial y del uso inapropiado de las vías vecinales para transporte diverso sin respetar el orden de diseño.

\section{SERVICIOS BÁSICOS:}

a. Agua para Consumo Humano: El agua para tal fin, proviene de dos manantiales de la parte media de la cuenca, el agua se entuba no se potabiliza, tampoco se administra el servicio, este llega gratuito como legado de W. R. Grace. No existen conexiones domiciliarias para los asentamientos humanos [8], se abastece a estas poblaciones con agua subterránea clasificada como de tipo I con presencia de coliformes totales.

b. Desague: No existe una red colectora de aguas residuales para domicilio ni industria, se utiliza el antiguo canal agrícola descubierto en varios tramos (atraviesa el centro urbano). c. Energía Eléctrica: El Gobierno local administra el servicio, con cobertura regular en todo el centro urbano.

d. Servicio de Recolección, Acopio y Tratamiento de Residuos Sólidos: Se abastece el servicio de barrido y recolección pero la disposición final es en botaderos. Crece la informalidad del servicio de recojo y los botaderos

e. clandestinos. Se recurre a la quema de restos de caña, el humo emanado afecta a 250 familias, con tendencia de crecimiento.

3. DINÁMICA POBLACIONAL: Descenso sostenido de la población desde 1981, por razones económicas y subversión. Se evidencia la concentración de la población en espacios reducidos. Densidad promedio actual 180 hab./ha.

4. VIVIENDA: Existen 25191 hab., distribuidos en 14 urbanizaciones y 8 asentamientos humanos. Predomina el uso casa habitación (95\%) y la consolidación del tipo de material. El 1952 W. R. Grace, planificó el uso del suelo urbano, pero se interrumpe con la reforma agraria y el paso a cooperativa, los nuevos propietarios alteran los diseños de las viviendas, incluso la línea de eje de las manzanas, no declarando las modificaciones y no pudiendo registrar las propiedades como suyas. El problema de la informalidad de la propiedad (invasiones paulatinas), se agudiza al no haberse efectuado un catastro.

5. EQUIPAMIENTO DEL SECTOR SALUD: Presencia del sector público y privado, con limitaciones de abastecimiento e insumos. Se adolece de falta de áreas verdes, sólo hay $2 \mathrm{~m}^{2}$ por habitante, pese a practicarse la Gran Industria. Urge hacer cumplir las recomendaciones del PAMA a AIPSA.

6. EQUIPAMIENTO DEL SECTOR EDUCACIÓN: No existe carencia de infraestructura, pero el nivel de enseñanza es bajo. Principal motor impulsor de la migración.

7. GESTIÓN MUNICIPAL: No se establece la directriz del distrito (Planes de Desarrollo), la clase política influye negativamente en la continuidad de procesos.

8. ACTIVIDADES ECONÓMICO PRODUCTIVAS: Cambio significativo de actividad predominante, de la agroindustria se pasa al comercio (formal e informal), las remesas presentan un crecimiento vertiginoso en los últimos cinco años; la agricultura individual tiende a desaparecer por falta de mercado.

\section{CONCLUSIONES.}

- El casco urbano de Paramonga creció desorganizadamente, se permitió el uso del suelo, sin prever las consecuencias. El efectuar una planificación territorial y enriquecerla con la normatividad vigente, permite generar una armonía entre el hombre y el espacio, condicionando el desarrollo sostenible.

- A través de la zonificación espacial, con proyección al 2015, se destina el uso de las islas rústicas para uso 
recreativo, taller y manufactura, servicios (formalización y organización del servicio de transporte), y vivienda (90 $000 \mathrm{~m}^{2}$ ).

- La adopción de las medidas mencionadas, permitirá satisfacer la demanda actual y futura de vivienda, evitando futuros conflictos sociales por demanda de espacios.

- Todo esto puede resumirse en la necesidad de consolidar un catastro, Ley Orgánica de Municipalidades № 27972, artículo 79ำ (competencia), y Resolución Ministerial № 150 - 2006 - vivienda, articulo 6응 (obligación).
Proyectos a desarrollarse con carácter de urgencia: Potabilización del Agua, Estudio Integral de la Red de Desagüe, Implementar un Plan de Desarrollo y Gestión Distrital, Dotar de equipamiento y medicinas a los Puestos de Salud y establecimiento de usos y funciones de las vía

\section{REFERENCIAS BIBLIOGRÁFICAS}

[1] AMAYA Pingo, Pedro. Guía Para la Identificación y Diseño de Proyectos de Desarrollo Local - 1. Lima. CONCYTEC \& UNFV. 2002. 103 p.

[2] ÁVILA Acosta, R.B. 2001. Metodología de la Investigación: Como Elaborar la Tesis y/o Investigación Ejemplos de Diseños de Investigación. Lima, Estudios y Ediciones R.A. 236 p.

[3] CAYCHO CHUMPITAZ, Hernán.1996. Paramonga: Apuntes Para una Caracterización Socioeconómica. Paramonga. Municipalidad de Paramonga.78 p.

[4] CENTRO DE ESTUDIOS REGIONALES ANDINOS BARTOLOMÉ DE LAS CASAS. 2001. La Red Urbana de la Región Cusco: Jerarquización de Centros Poblados y su Aplicación en la Asignación de Inversiones en el Territorio. Cusco. 119p.

[5] CENTRO DE ESTUDIOS REGIONALES ANDINOS BARTOLOMÉ DE LAS CASAS. 1998. Plan de Ordenamiento Urbano de Acomayo. Oficina Regional Técnica de Apoyo Municipal. Cusco. 130p.

[6] DUEÑAS A., DEFILIPPI M., RAMÍREZ H., GARCÍA V. \& VELEZ DE VILLA M. "Agua y Azúcar: Consecuencias Ambientales y Económicas de su Gestión en la Industria". Yachay Yacu № 08 (03): 42-50, Mayo 2006.

[7] ESSALUD. Resultados del Informe Anual de la Posta Médica de EsSalud Belén. 2006. Paramonga.

[8] Hospital Santa Rosa. Resultados del Informe Anual del Hospital Santa Rosa. 2006. Paramonga.

[9] INSTITUTO NACIONAL DE DESARROLLO URBANO. 1991. Plan de Ordenamiento Urbano de Paramonga. Paramonga. 42p.

[10] INSTITUTO NACIONAL DE ESTADÍSTICA E INFORMÁTICA. 2006. Censos Nacionales: $X$ de Población y V de Vivienda. [CD-ROM]. Aplicación de Redatam. Versión 1.0. Lima. INEl. Programa Computacional.

[11] INSTITUTO NACIONAL DE RECURSOS NATURALES. 2005. Inventario de Fuentes de Agua Subterránea en el Valle Fortaleza. Lima. $120 \mathrm{p}$.

[12] MUNICIPALIDAD DISTRITAL DE PARAMONGA. Resultados del Estudio Socioeconómico del Distrito de Paramonga - Informe № 07-96-DSS-MDP. \& Informe № 23-06-DSS-JTP. Paramonga.

E-mail: betto_huiman@hotmail.com 